\title{
Orientações sobre aleitamento materno em consultas de pré-natal
}

Recebido em: 09/10/2013

Aprovado em: 14/06/2014

\author{
Gardenia Gomes Pereira \\ Mariana Cavalcante Martins ${ }^{2}$ \\ Emilly Karoline Freire Oliveira ${ }^{3}$ \\ Denise Maia Alves da Silva ${ }^{4}$ \\ Ana Debora Assis Moura
}

Resumo: Objetivou-se avaliar a atuação do enfermeiro na consulta de pré-natal quanto às orientações sobre aleitamento materno. Pesquisa observacional não participante, quantitativa, realizada em três Centros de Saúde da Família de Fortaleza-CE, Brasil, durante atendimento a 54 gestantes, utilizando um checklist. Das 54(100\%) consultas observadas, verificou-se percentual reduzido em relação às orientações: anatomia da mama $1(1,9 \%)$, posição para amamentar $5(9,3 \%)$ e pega adequada $18(33,3 \%)$; em contrapartida o preparo da mama 35(64,8\%) e duração do aleitamento 40(74,1\%), foram mais orientados. Identificaram-se lacunas que devem ser minimizadas por meio da corresponsabilidade dos profissionais referente à prática do aleitamento materno.

Descritores: Aleitamento materno; enfermagem; cuidado pré-natal.

\section{Guidelines on breastfeeding in prenatal consultations}

Abstract: The objective was to assess the performance of nurses in prenatal consultation regarding the guidelines on breastfeeding. Quantitative observational non-participant research carried out in three Family Health Centers of Fortaleza-CE, Brazil, during the assistance to 54 pregnant women through a checklist. Of the $54(100 \%)$ consultations observed, there was a low percentage on the following guidelines: breast anatomy 1 (1.9\%), proper position 5 (9.3\%) and proper grasp 18 (33.3\%); however, breast preparation $35(64.8 \%)$ and duration of breastfeeding $40(74.1 \%)$ were more oriented. We identified gaps that should be minimized through the professionals' corresponsibility on the promotion of breastfeeding.

Descriptors: Breast Feeding, Nursing, Prenatal Care.

\section{Orientaciones acerca de la lactancia materna en las consultas de prenatal}

Resumen: El objetivo fue evaluar la actuación del enfermero en la consulta de prenatal cuanto a las orientaciones sobre lactancia materna. Investigación observacional no participante, en tres Centros de Salud Familiar de Fortaleza-CE, Brasil, durante atención a 54 mujeres embarazadas, utilizándose lista de verificación. De las $54(100 \%)$ consultas observadas, hubo bajo porcentaje en las siguientes orientaciones: anatomía de la mama 1(1,9\%), posición para amamantar 5 (9, 3\%) y pega 18 (33,3\%); en contradicción, preparación de la mama $35(64,8 \%)$ y duración de la lactancia 40 (74,1\%), fueron más orientados. Se identificaron lagunas que deben ser minimizados a través de la responsabilidad de los profesionales en la promoción de la lactancia materna.

Descriptores: Lactancia Maternal, Enfermería, Atención Prenatal.

\section{INTRODUÇÃO}

númeras são as vantagens do Aleitamento Materno (AM) para mãe, bebê, família e sociedade. Para a mãe, facilita a involução uterina, reduz o risco de hemorragia, promove o vínculo afetivo, permite o prazer de amamentar, contribui para o retorno ao peso pré-gravídico, previne osteoporose; e para o bebê, é um alimento completo, facilita a eliminação de mecônio e icterícia, protege contra infecções, promove o vínculo afetivo, diminui as chances de desenvolver alergias; para família e sociedade, é livre de impurezas, pronto, na temperatura adequada, diminui as internações e custos e é gratuito ${ }^{(1)}$.

A forma mais eficaz, segura e completa de obter crescimento e desenvolvimento adequados de uma criança é garantir o AM exclusivo até o sexto mês de vida, sendo essa prática o padrão ouro para lactentes nessa faixa etária ${ }^{(2)}$. Embora existam metas e compromissos dos programas e das políticas governamentais brasileiras, juntamente com recomendações das Nações Unidas, o desmame precoce continua sendo um grave problema de saúde pública ${ }^{(3)}$.

É válido ressaltar que o desmame precoce ainda atinge proporções elevadas, a prevalência do Aleitamento Materno Exclusivo(AME) em menores de seis meses foi de $41 \%$ no conjunto das capitais brasileiras, dentre todas as regiões a norte com $45,9 \%$ apresentou a melhor situação e a nordeste com 37,0\% apresentou a pior situação de $\mathrm{AM}^{2}$.

Em decorrência desses dados, a Estratégia Saúde da Família vem se consolidando como um dos eixos estruturantes do Sistema Único de Saúde (SUS), por meio de um movimento de expressiva expansão de cobertura populacional, aprimorando em muito o acesso da população às ações de saúde ${ }^{(2)}$.

Nesse contexto, o enfermeiro é o profissional que mais estreitamente se relaciona com a mulher durante o ciclo gravídicopuerperal e tem importante papel nos programas de educação em saúde. Desse modo, o diálogo franco, a sensibilidade e a capacidade de percepção de quem acompanha o pré-natal são condições primordiais para que o saber em saúde seja posto à disposição da mulher e família(1).

Ao ponderar esse pressuposto, é preconizado que se tenha a oferta de ações educativas que viabilizem a mulher sobre o conhecimento do corpo, dentre outros aspectos. Estas ações devem, preferencialmente, ser realizadas por meio de metodologias participativas, para que o conhecimento prévio das mulheres possa ser intercambiado dentro dos grupos existentes nos serviços de saúde ${ }^{(4)}$.

Diante de inúmeros motivos que levam ao desmame precoce: trabalho materno fora de casa, oferta de bicos ou chupetas, atendimento puerperal efetuado no serviço privado e primiparidade ${ }^{(5),(6)}$;

'Enfermeira graduada pela Faculdade Integrada da Grande Fortaleza. Fortaleza, CE, Brasil. E-mail: gardenia_lucas@hotmail.com

${ }^{2}$ Enfermeira. Doutora em Enfermagem. Docente da Graduação em Enfermagem da Universidade Federal do Ceará (UFC). Fortaleza, CE, Brasil.

${ }^{3}$ Enfermeira. Mestranda em Enfermagem da Universidade Federal do Ceará (UFC). Fortaleza, CE, Brasil.

${ }^{4}$ Enfermeira. Mestre em Saúde Coletiva. Docente da Graduação em Enfermagem da Faculdade Integrada da Grande Fortaleza- FGF. Fortaleza, CE, Brasil.

${ }^{5}$ Enfermeira. Mestre em Enfermagem. Doutoranda em Saúde Publica pela Universidad de Ciencias Empresariales y Sociales 
introdução precoce da alimentação(7), dentre outros, uma pesquisa ${ }^{(8)}$ que objetivou investigar a atuação dos enfermeiros nas atividades de assistência, promoção e incentivo ao $\mathrm{AME}$, evidenciou a importância da atuação do enfermeiro no $A M E$, havendo ainda a necessidade da sensibilização para mudança de comportamento dos profissionais.

Assim, partindo desse contexto que decidiu-se analisar por meio observacional, a atuação dos enfermeiros com enfoque no AM, não antes pesquisado. $O$ estudo objetivou avaliar consultas de pré-natal realizadas por enfermeiros(as) com enfoque no AM em Centros de Saúde da Familia (CSF), no município de Fortaleza-CE.

\section{MÉTODOS}

Tratou-se de pesquisa observacional, não participante, estruturada, com abordagem quantitativa. Foi realizada em três Centros de Saúde da Família (CSF), localizados na periferia de Fortaleza-CE.

Observou-se 54 consultas de pré-natal realizadas por enfermeiras que atenderam aos critérios: enfermeiras que estivessem exercendo atividades no CSF, no momento da coleta e que realizassem consulta de pré-natal. Ressalta-se que não foi estabelecido período gestacional, pois o Ministério da Saúde preconiza que em todas as consultas se deve enfatizar a importância do $\mathrm{AM}^{(4)}$. Na sequência, os enfermeiros foram contactados e esclarecidos quanto à pesquisa, ao período de coleta e aos direitos como participantes.

A coleta de dados foi realizada por meio de observação não participante, mediante visitas semanais, durante os dias de consultas de pré-natal de cada profissional; utilizou-se formulário do tipo checklist, previamente elaborado pelas pesquisadoras envolvidas, dividido em duas etapas: caracterização dos profissionais; e aspectos relacionados ao AM que deveriam ser explanados pelo profissional durante a consulta, abordando os temas: conhecimento prévio da gestante sobre amamentação/AM, principais tabus existentes, anatomia da mama, realização do exame físico das mamas, fisiologia da lactação, preparo das mamas para o aleitamento, vantagens da amamentação para a mulher, criança, família e sociedade, manejo da amamentação (posição e pega), duração da amamentação e oportunidade da gestante trocar experiência, por meio de diálogo no momento das consultas e reuniões grupais. Salienta-se, todavia, que se realizou um pré-teste com observação de dez gestantes, sendo estas não participantes da presente pesquisa, com intuito de aperfeiçoar o checklist. Vale destacar que esses temas foram embasados no Manual Técnico: Pré-Natal e Puérperio - Atenção Qualificada e Humanizada(1).

Os dados foram tabulados e quantificados com auxílio do Programa Statistical Package for the Social Sciences (SPSS) versão 19.0. Para a análise, valeu-se da estatística descritiva, por meio de frequências absolutas e relativas, organizados em tabelas.

A pesquisa foi aprovada pelo Comitê de Ética em Pesquisa da Universidade de Fortaleza, segundo o protocolo de $n^{\circ} 236 / 10$. Foram respeitadas todas as recomendações e requisitos éticos previstos para pesquisas envolvendo seres humanos, conforme Resolução $n^{\circ} 196 / 96^{(9)}$.

\section{RESULTADOS}

No tocante à distribuição das orientações das enfermeiras é expresso e pode ser vizualizado no decorrer da tabela 1, que com relação à mama, das 54 consultas observadas, somente uma $(1,9 \%)$ gestante foi orientada em relação à anatomia da mama e duas $(3,7 \%)$ acerca da fisiologia da mama. Ademais, trinta e quatro (63\%) gestantes foram submetidas ao exame físico da mama.

No que diz respeito às orientações sobre as vantagens do AM, pode-se observar que as mesmas foram incipientes, pois somente quatro $(7,4 \%)$ gestantes foram orientadas quanto às vantagens do aleitar para mulher, $24(44,4 \%)$ para criança, quatro $(7,4 \%)$ para família, uma (19\%) para sociedade. Verificou-se ainda que somente seis $(11,1 \%)$ gestantes foram orientadas sobre as desvantagens do desmame precoce para a criança .

Acerca das orientações envolvendo os aspectos da prática da amamentação, somente cinco (9,3\%) gestantes foram orientadas sobre a posição adequada; a pega correta foi ressaltada em 18 (33,3\%) consultas e o preparo da mama em 35 (64,8\%). Em relação à duração do $\mathrm{AM}, 40$ (74,1\%) gestantes receberam orientações.

Tabela 1 - Distribuição das orientações, durante a consulta de pré-natal realizada por enfermeiros. Fortaleza, CE, Brasil, 2010.

\begin{tabular}{|c|c|c|c|c|}
\hline & \multicolumn{2}{|c|}{ Sim } & \multicolumn{2}{|c|}{ Não } \\
\hline \multicolumn{5}{|c|}{ Orientações com relação à mama } \\
\hline & $\mathbf{N}$ & $\%$ & $\mathbf{N}$ & $\%$ \\
\hline $\begin{array}{l}\text { Anatomia } \\
\text { da mama }\end{array}$ & 1 & 1,9 & 53 & 98,1 \\
\hline Exame físico & 34 & 63 & 20 & 37 \\
\hline Fisiologia & 2 & 3,7 & 52 & 93,3 \\
\hline \multicolumn{5}{|c|}{ Orientações sobre as vantagens do aleitamento materno } \\
\hline & $\mathbf{N}$ & $\%$ & $\mathbf{N}$ & $\%$ \\
\hline $\begin{array}{l}\text { Vantagens } \\
\text { Mulher }\end{array}$ & 4 & 7,4 & 50 & 92,6 \\
\hline $\begin{array}{l}\text { Vantagens } \\
\text { Criança }\end{array}$ & 24 & 44,4 & 30 & 55,6 \\
\hline $\begin{array}{l}\text { Vantagens } \\
\text { Família }\end{array}$ & 4 & 7,4 & 50 & 92,6 \\
\hline $\begin{array}{l}\text { Vantagens } \\
\text { Sociedade }\end{array}$ & 1 & 1,9 & 53 & 98,1 \\
\hline Desvantagens & 6 & 11,1 & 48 & 88,9 \\
\hline \multicolumn{5}{|c|}{ Orientações sobre a amamentação } \\
\hline & $\mathbf{N}$ & $\%$ & $\mathbf{N}$ & $\%$ \\
\hline $\begin{array}{l}\text { Posição } \\
\text { adequada }\end{array}$ & 5 & 9,3 & 49 & 90,7 \\
\hline Pega correta & 18 & 33,3 & 36 & 66,7 \\
\hline $\begin{array}{l}\text { Preparo da } \\
\text { mama }\end{array}$ & 35 & 64,8 & 19 & 35,2 \\
\hline $\begin{array}{l}\text { Duração do } \\
\text { aleitamento }\end{array}$ & 40 & 74,1 & 14 & 25,9 \\
\hline
\end{tabular}

${ }^{*} \mathrm{~N}=54$, se refere ao número de consultas que foram observadas.

Ao observar a tabela 2, pode-se salientar que foi detectado, também, que não houve diálogo entre o profissional e a gestante, impossibilitando 35 (64,8\%) gestantes de exporem conhecimentos; 48 (88,9\%) não evidenciaram tabus, pois as demais não foram estigadas e (100\%) não tiveram oportunidade de trocar experiência, por meio de diálogo e/ou grupo de gestantes. 
Tabela 2 - Distribuição da possibilidade de diálogo entre gestante e enfermeiro sobre a prática do aleitamento materno durante a consulta de pré-natal realizada por enfermeiros. Fortaleza, CE, Brasil, 2010

\begin{tabular}{ccccc} 
& \multicolumn{2}{c}{ Sim } & \multicolumn{2}{c}{ Näo } \\
& \multicolumn{2}{c}{ Frequência de diálogo } & \\
\hline & $\mathbf{N}$ & $\%$ & $\mathbf{N}$ & $\%$ \\
\hline $\begin{array}{c}\text { Conhecimento } \\
\text { das } \\
\text { gestantes }\end{array}$ & 19 & 35,2 & 35 & 64,8 \\
\hline $\begin{array}{c}\text { Tabus } \\
\text { Trocas de }\end{array}$ & 6 & 11,1 & 48 & 88,9 \\
\hline experiência & - & - & 54 & 100 \\
\hline
\end{tabular}

\section{DISCUSSÃO}

Torna-se essencial que a gestante conheça o processo de aleitar, desde da produção de leite até o ato de amamentar, para que haja empoderamento e, consequentemente, reflexão crítica, favorecendo, assim, a desmitificação de possíveis tabus que impedem tal prática ${ }^{(10)}$. Assim, conhecer a anatomia e a fisiologia da mama é fundamental para o aconselhamento e a resolução de problemas, pois, dessa forma, a mulher, conhecendo o processo que circunda o ato de amamentar, poderá decidir sobre a duração do AM.

Acredita-se que a orientação acerca do AM deva ser reforçada pelos enfermeiros e demais profissionais das Unidades de Saúde, particularmente no decorrer do pré-natal, puerpério e nas visitas domiciliares, garantindo que a informação chegue adequadamente às mulheres com relação à manutenção da lactação. A técnica da pega correta, o intervalo entre as mamadas, os direitos trabalhistas, a alimentação, as orientações de como conciliar a amamentação com a vida pessoal, profissional e com seus projetos de vida, assim como os benefícios para a saúde materna e a relação de proteção para o câncer de mama são alguns dos temas que devem ser abordados ${ }^{11}$.

Um estudo realizado em Alfenas-MG ${ }^{11}$, mostrou que as mães orientadas acerca dos benefícios do $A M$, continuavam a amamentar e quando indagadas sobre os motivos de continuidade, citaram as vantagens do $\mathrm{AM}$, dentre elas as mais faladas: "praticidade", "menor custo", "perda de peso", "satisfação", "prevenção do câncer de mama", "recuperação pós-parto mais rápida" e "estética". O que nos remete que a importância de orientações durante as consultas pré-natais favorece a prática de aleitar.

Com relação aos benefícios para o filho, ressalta-se que no Brasil, ainda, são poucos os estudos que abordem a relação do AM e sobrepeso e obesidade infantil ${ }^{12}$. Entretanto, destaca-se que crianças amamentadas representam fator de proteção, indicando ainda que o fator de risco para essa condição é a ausência do $\mathrm{AM}^{13}$.

No que concerne aos aspectos mais práticos do ato de amamentar, observou-se que poucas gestantes foram orientadas sobre a posição adequada, bem como a pega correta. Em contrapartida com os achados, pesquisa ${ }^{14}$ realizada, detectou-se que o melhor desempenho dos enfermeiros foi em relação à técnica correta da amamentação, conhecimentos gerais sobre leite materno, cuidados e manejos com as mamas e respectivos problemas.
A duração mediana do AM no Brasil aumentou cerca de 1,5 mês, passando de 295,9 dias, em 1999, para 341,6 dias, em 2008, apesar do incremento verificado na manutenção do AM no país, sua mediana continua inferior à observada na Bolívia (17,5 meses), Guatemala (19,9 meses) e Peru (19,5 meses) na década de $1990^{(15)}$.

Evidenciou-se, ainda, que nenhuma das gestantes atendidas dialogou com as enfermeiras, sendo somente receptoras de informações, corroborando com achados de pesquisa que detectou que $42 \%$ das gestantes relataram sentimento de abandono e falta de apoio das unidades básicas, tendo como consequência o insucesso da amamentação(16).

Vale frisar, também, que a causa determinante do desmame precoce refere-se a tabus estabelecidos pela sociedade: leite fraco, leite secou, leite insuficiente e as mamas caírem; tabus estes que poderiam ser desmitificados por meio do diálogo entre profissional e família( ${ }^{(17)}$.

Partindo deste pressuposto, compreende-se que o enfermeiro enquanto profissional que atua diretamente com este cliente deva ser agente ativo de transformação do contexto social da área sob sua responsabilidade, assim como estar desenvolvendo práticas que respeitem a dialógica entre os sujeitos, a dignidade das gestantes e que garanta seus direitos como cidadãs, usuárias do sistema de saúde brasileiro, além de permitir que as mulheres em período gestacional possam esclarecer suas dúvidas, minimizar seus anseios e tenham um acompanhamento pré-natal satisfatório, de modo que suas necessidades de saúde sejam respondidas ${ }^{(18)}$.

A interrupção do ato de amamentar pode estar principalmente relacionado ao reduzido conhecimento das puérperas acerca do manejo da lactação. Logo, torna-se relevante salientar a necessidade de orientação e informação sobre medidas preventivas para a manutenção da amamentação e a promoção da saúde da mulher, ainda durante as consultas de pré-natal ou mesmo para todas as mulheres em idade fértil, no planejamento familiar ou em outras oprtunidades, a fim de suprir dúvidas e quebrar tabus relacionados à amamentação(11).

Assim, diante de todo esse contexto, infere-se que as orientações não estão sendo realizadas de acordo com o preconizado pelo Ministério da Saúde; apesar do aumento da cobertura de consultas de pré-natal, não há garantia de qualidade de consultas ${ }^{(19)}$.

\section{CONCLUSÕES}

Diversos estudos enfocam as causas do desmame diante da visão materna, bem como do profissional; porém, este estudo observacional verificou que as orientações dispensadas são deficientes, baseado no que é preconizado.

Para prestar assistência de forma integral, a equipe de enfermagem deve trabalhar de forma interdisciplinar, utilizando da comunicação como instrumento de trabalho nesse processo - aleitamento materno versus família.

Destarte, faz-se mister enfatizar a necessidade de que haja um empoderamento das mães/familiares, a partir da comunicação e troca de conhecimentos, tornando-as "protagonistas" deste enrredo e facilitando o repasse das informações referentes ao AM, forjando assim, uma comunidade mais autonoma. 


\section{Referências}

1. Ministério da Saúde (BR). Secretaria de Atenção à Saúde. Prénatal e Puerpério: atenção qualificada e humanizada - manual técnico. Brasília: Ministério da Saúde; 2006.

2. Ministério da Saúde (BR). Secretaria de Atenção à Saúde. Saúde da criança: nutrição infantil aleitamento materno e alimentação complementar. Brasília (DF); 2009.

3. Caminha MFC, Serva VB, Arruda IKG, Batista Filho M, Caminha MFC. Aspectos históricos, científicos, socioeconômicos e institucionais do aleitamento materno. Rev Bras Saúde Mater Infant. 2010; 10(1):25-37.

4. Rios CTF, Vieira NFC. Ações educativas no pré-natal: reflexão sobre a consulta de enfermagem como um espaço para educação em saúde. Ciênc Saúde Coletiva. 2007; 12(2):477-86.

5. Salustiano LPQ, Diniz ALD, Abdallah VOS, Pinto RMC. Fatores associados à duração do aleitamento materno em crianças menores de seis meses. Rev. Bras. Ginecol. Obstet. [online]. 2012; 34(1): 28-33.

6. Leone CR, Sadeck LSR. Fatores de risco associados ao desmame em crianças até seis meses de idade no município de São Paulo. Rev. paul. pediatr. [online]. 2012; 30(1): 21-26.

7. Palmeira PA, Santos SMC, Vianna RPT. Prática alimentar entre crianças menores de dois anos de idade residentes em municípios do semiárido do Estado da Paraíba, Brasil. Rev. Nutr. [online]. 2011; 24(4):553-563.

8. Capeleto SM, Domingues ACP, Silva DA, Filho PLS. Atuação do enfermeiro na prática do aleitamento materno exclusivo nas unidades de saúde da família. REMENFE. 2010; 1-15.

9. Ministério da Saúde (BR). Conselho Nacional de Saúde. Resolução 196, de 10 de outubro de 1996: diretrizes e normas regulamentadoras de pesquisa envolvendo seres humanos. Brasília: Ministério da Saúde; 1996.

10. Faleiros FTV, Trezza EMC, Carandina L. Aleitamento materno: fatores de influência na sua decisão e duração. Rev Nutr Campinas. 2008; 19(5):623-30.
11. Gradim CVC, Magalhães MC, Faria MCF, Arantes CIS. Aleitamento materno como fator de proteção para o câncer de mama. Rev Rene. 2011; 12(2): 358-64.

12. Simon VGN, Souza JMP, Souza SB. Aleitamento materno, alimentação complementar, sobrepeso e obesidade em préescolares. Rev Saúde Pública. 2009; 43(1):60-9.

13. Ferreira HS, Vieira EDF, Cabral Júnior CR, Queiroz MDR. Aleitamento Materno por trinta ou mais dias é fator de proteção contra sobrepeso em pré-escolares da região semiárida de Alagoas. Rev Assoc Med Bras. 2010; 56(1):74-80.

14. Caldeira AP, Aguiar GN, Magalhães WAC, Fagundes GC. Conhecimentos e práticas de promoção do aleitamento materno em Equipes de Saúde da Família em Montes Claros, Minas Gerais, Brasil. Cad Saúde Pública. 2007; 23(8):1965-70.

15. Venancio SI, Escuder MML, Saldiva SRDM, Giugliani ERJ. A prática do aleitamento materno nas capitais brasileiras e Distrito Federal; situação atual e avanços. J Pediatr. 2010; 86(4):317-24.

16. Oliveira MIC, Souza IEO, Santos EM, Camacho LAB. Avaliação de apoio recebido para amamentar: significados de mulheres usuárias de unidades básicas de saúde do Estado do Rio de Janeiro. Ciênc Saúde Coletiva. 2010; 15(2):599-608.

17. Azeredo CM, Maia TM, Rosa TCA, Silva FF, Cecon PR, Cotta RMM. Percepções de mães e profissionais de saúde sobre aleitamento materno: encontros e desencontros. Rev Paul Pediatr. 2008; 26(4):336-44.

18. Melo RM, Brito RS, Carvalho FPB, Pessoa Júnior JM, Barros SDOL. A integralidade da assistência no contexto da atenção pré-natal. Rev Rene. 2011; 12(4):750-7.

19. Tavares MC, Aires JS, Dodt RCM, Joventino ES, Oriá MOB, Ximenes LB. Application of Breastfeeding Self-Efficacy Scale-Short Form to post-partum women in rooming-in care: a descriptive study. Online Braz J Nurs. [online]. 2010; 9(1). 